\title{
Corrigendum: Effects of Zinc Oxide Nanoparticles Synthesized Using Aspergillus niger on Carbapenem- Resistant Klebsiella pneumonia In Vitro and In Vivo
}

\begin{abstract}
Elsayim Rasha ${ }^{1 *}$, Manal M. Alkhulaifi ${ }^{1}$, Monerah AlOthman ${ }^{1}$, Ibrahim Khalid ${ }^{2}$, Elnagar Doaa ${ }^{2}$, Khatab Alaa ${ }^{2}$, Manal A. Awad ${ }^{3}$ and Mohnad Abdalla ${ }^{4 *}$

${ }^{1}$ Department of Botany and Microbiology, College of Science, King Saud University, Riyadh, Saudi Arabia, ${ }^{2}$ Department of Zoology, College of Science, King Saud University, Riyadh, Saudi Arabia, ${ }^{3}$ College of Science, King Saud University, Riyadh, Saudi Arabia, ${ }^{4}$ Department of Medicine, Vascular Biology Center, Medical College of Georgia at Augusta University, Augusta, GA, United States
\end{abstract}

Keywords: zinc oxide nanoparticle, antimicrobial resistance, biological synthesis, carbapenem-resistant Klebsiella pneumoniae (KPC), Aspergillus niger, wound recovery

\section{A Corrigendum on:}

Effects of Zinc Oxide Nanoparticles Synthesized Using Aspergillus niger on CarbapenemResistant Klebsiella pneumonia In Vitro and In Vivo by Rasha E, Alkhulaifi MM, AlOthman M, Khalid I, Doaa E, Alaa K, Awad MA and Abdalla M (2021). Front. Cell. Infect. Microbiol. 11:748739. doi: 10.3389/fcimb.2021.748739

\section{INCORRECT AFFILIATION}

a section of the journ

Frontiers in Cellular and

Infection Microbiology

Received: 26 December 2021

Accepted: 21 January 2022

Published: 10 February 2022

Citation:

Rasha E, Alkhulaifi MM, AlOthman M, Khalid I, Doaa E, Alaa K, Awad MA and Abdalla M (2022) Corrigendum: Effects of Zinc Oxide Nanoparticles Synthesized Using Aspergillus niger on Carbapenem-Resistant Klebsiella pneumonia In Vitro and In Vivo. Front. Cell. Infect. Microbiol. 12:843577. doi: $10.3389 /$ fcimb.2022.843577
In the published article, there was an error in affiliation 3. Instead of "King Abdullah Institute of Nanotechnology, King Saud University, Riyadh, Saudi Arabia", it should be "College of Science, King Saud University, Riyadh, Saudi Arabia”.

The authors apologize for this error and state that this does not change the scientific conclusions of the article in any way. The original article has been updated.

Publisher's Note: All claims expressed in this article are solely those of the authors and do not necessarily represent those of their affiliated organizations, or those of the publisher, the editors and the reviewers. Any product that may be evaluated in this article, or claim that may be made by its manufacturer, is not guaranteed or endorsed by the publisher.

Copyright (c) 2022 Rasha, Alkhulaifi, AlOthman, Khalid, Doaa, Alaa, Awad and Abdalla. This is an open-access article distributed under the terms of the Creative Commons Attribution License (CC BY). The use, distribution or reproduction in other forums is permitted, provided the original author(s) and the copyright owner(s) are credited and that the original publication in this journal is cited, in accordance with accepted academic practice. No use, distribution or reproduction is permitted which does not comply with these terms. 